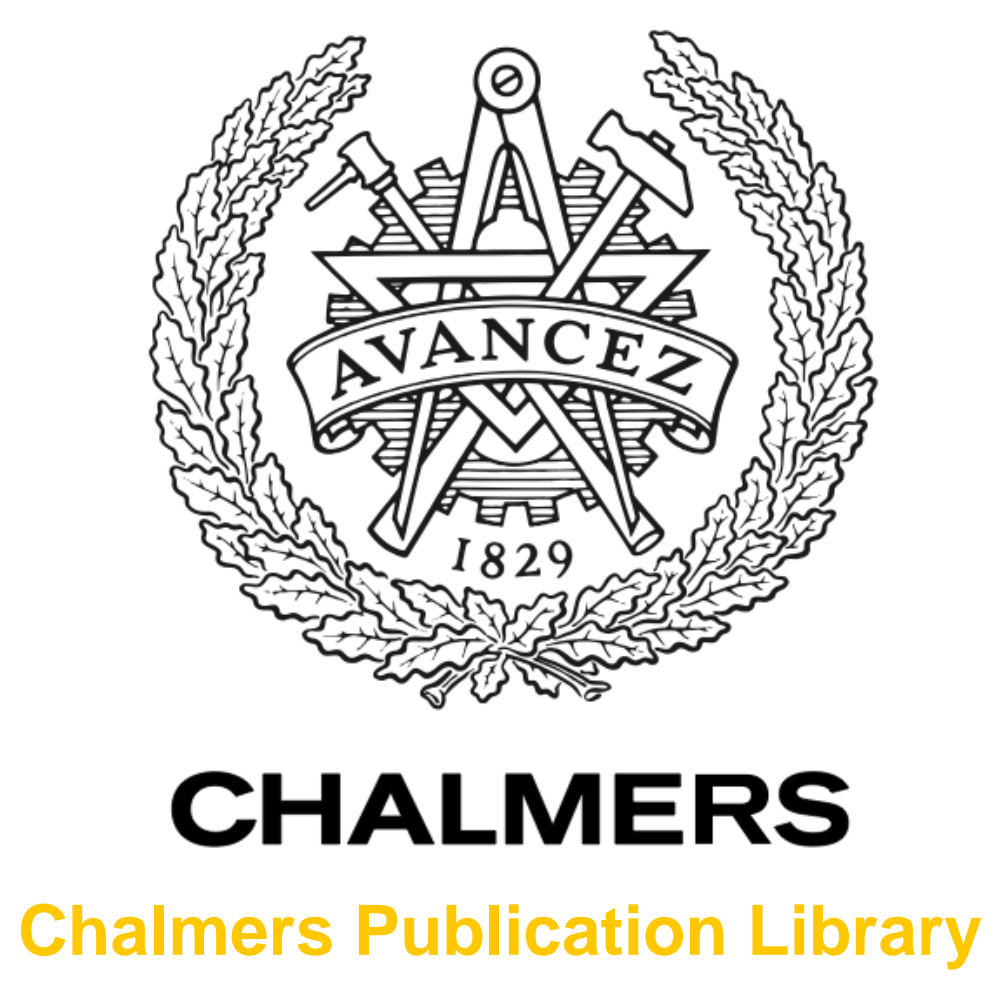

A microwave measurement system for stroke detection

This document has been downloaded from Chalmers Publication Library (CPL). It is the author's version of a work that was accepted for publication in:

7th Loughborough Antennas and Propagation Conference, LAPC 2011, Loughborough, 14 November through 15 November 2011

Citation for the published paper:

Fhager, A. ; Persson, M. (2011) "A microwave measurement system for stroke detection". 7th Loughborough Antennas and Propagation Conference, LAPC 2011, Loughborough, 14

November through 15 November 2011

http://dx.doi.org/10.1109/LAPC.2011.6114001

Downloaded from: http://publications.lib.chalmers.se/publication/155744

Notice: Changes introduced as a result of publishing processes such as copy-editing and formatting may not be reflected in this document. For a definitive version of this work, please refer to the published source. Please note that access to the published version might require a subscription. 


\title{
A Microwave Measurement System for Stroke Detection
}

\author{
Andreas Fhager ${ }^{\# 1}$, Mikael Persson ${ }^{\# 2}$ \\ ${ }^{*}$ Department of Signals and Systems, Chalmers University of Technology \\ SE-41296 Gothenburg, Sweden \\ ${ }^{1}$ andreas. fhagerechalmers.se \\ ${ }^{2}$ mikael.perssonechalmers.se
}

\begin{abstract}
In this paper we describe a microwave based measurement setup and a signal processing algorithm for stroke detection and diagnostics. We conclude that the technique is feasible and clinical studies show promising results and encourage further investigations.
\end{abstract}

\section{INTRODUCTION}

With a population of a little more than 9 millions, roughly 30000 people suffer a stroke each year in Sweden. About 15$30 \%$ of them become permanently disabled, [1]. In the United States the number of strokes per year is approximately 700 000. Approximately $85 \%$ of all strokes are ischemic (blood clot induced) and 15\% hemorrhagic (bleeding) strokes. Stroke is placed third among reasons for acute death, and first among reasons for neurological dysfunction. Stroke is also resulting in most days of hospital nursing and therefore the most costly disease within the western world health care. The total social cost resulting from stroke in Sweden is estimated to EUR 1.5 billion per year. Among stroke survivors, 20\% have serious remaining dysfunctions, [1]. A much larger proportion has less conspicuous dysfunctions which still seriously affect quality of life for the patient and relatives.

Early clot resolving (thrombolytic) treatment has proved very successful for ischemic stroke patients. Accurate diagnostic procedures are however requires because if given to hemorrhagic stroke patients this treatment could instead be lethal. The effect of the thrombolytic treatment is better the sooner it is given and it cannot be given later than 4.5 hours since start of the symptoms, as the tissue damage at this time result in a high risk for bleeding, [2]. Today computer tomography $(\mathrm{CT})$ and sometimes magnetic resonance imaging (MRI) are used to diagnose the ischemic from the hemorrhagic strokes. The examinations in these facilities are however time consuming and together with transportation and other procedures in the chain of care the result is that only a minor portion if the ischemic stroke patients who could potentially benefit from the treatment actually receives it.

Microwave technology is a strong and novel candidate to fulfill the needed gap of a diagnostic method in the care of acute stroke patients. It has high sensitivity to differentiate between blood and brain tissue due to the high difference in permittivity, [3]. It is also a technology with components that can be made compact and thus be implemented earlier in the health care chain, in emergency rooms and in ambulances. The same microwave technology has also attracted extensive interest in the research community as a novel method for breast cancer detection as well as several other applications that are under investigation.

Previously we have made a numerical feasibility study of the possibility to detect a blood clot inside the brain, [4]. We have also made an experimental study using tissue like phantom material. Both these studies have shown that the proposed microwave technique could be a viable method to detect bleedings. Numerical studies have also been presented elsewhere, [5], [6]. We have performed a clinical study on 20 stroke patients of which 10 have a bleeding stroke and 10 a blood clot induced stroke. For comparison we also make measurements on healthy volunteers. Currently we are running a new study on 30 patients. The studies are made in collaboration with the Sahlgrenska University Hospital and the company Medfield Diagnostics AB. All clinical trials are approved by an Ethics Committee and the Medical Products Agency in Sweden. The measurements on the patients are made after they have gone through the normal diagnostic procedures and received treatment in order not to put the patient under any additional risk by interfering during the acute phase.

\section{MEASUREMENT PROTOTYPE}

The measurement strategy in this application is similar to what is traditionally used in microwave tomography applications. A number of transmitting and receiving antennas are mounted in a configuration surrounding the target object under investigation, in this case the skull. Data is measured using every possible combination of antennas as transmitter and receiver. For the measurements we use 10 triangular patch antennas with a V-shaped slot and a short-circuiting wall between the patch and the ground plane. The 10 antennas can be seen mounted inside a normal bicycle helmet in Fig. 1.

It is also necessary to use a matching medium between the antennas and the skull to couple the signal into the skull. A gap of air between the antenna and the skull could be disastrous to the development of a successful stroke detection 
application. A significant reflection would occur in the interface between the skin and the air due to the large

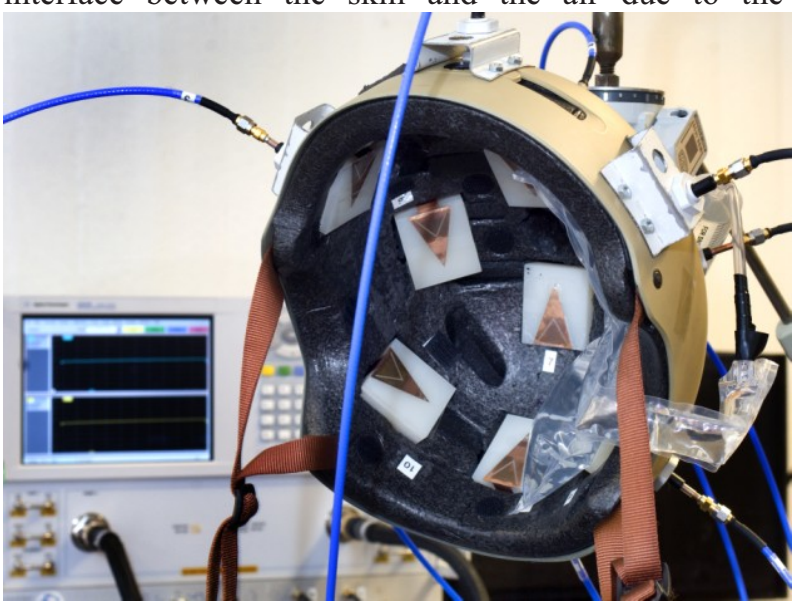

Figure 1. A photograph of the prototype microwave helmet. The patch antennas can be seen mounted inside a bicycle helmet.

difference in impedances. In breast cancer microwave tomography it is common to immerse the antennas and also the breast in some matching liquid before the measurements are made. For obvious reasons it is not a viable solution to submerge the skull of the stroke patient into a matching medium before the measurements. In this study we instead used plastic bags filled with the matching liquid. The bags were attached inside the helmet, covering the antennas. A system for adjusting the amount of liquid in the bags was used in order to adjust the fitting of the helmet on the patient, to accommodate different sizes of the skull and to make sure that the space between the antennas and the skull was completely filled with the matching liquid. The matching liquid bags are however not shown in the photo in Fig. \ref \{fig:helmet\}.

The antennas are designed to have the desired property in the vicinity of the lossy media in form of the skull. However the characteristics of the antennas are sensitive and changing with the surrounding media. They have a resonance frequency of about $1.3 \mathrm{GHz}$ in the actual measurement situation. Data in the frequency range $0.1 \mathrm{GHz}$ to $3.0 \mathrm{GHz}$ is collected using a network analyzer.

\section{SIGNAL PROCESSING}

In order to interpret the measured data and to make the diagnosis it is necessary to use some signal processing algorithm. We are currently investigating two different types of algorithms. One is a full dielectric reconstruction using inverse algorithms from microwave tomography, [7]. The other is a classifier based on calculated subspace distances in the measured data and a training set of data from patients with known diagnosis, [8]

Most of our work is however focused on using the classifier algorithm where we attempt to identify and separate the hemorrhagic stroke patients from the healthy and the ischemic stroke patients. Classifiers are based on some metric measures to separate the different classes. The situation here is that we have data from a large number of healthy patients but relatively fewer patients with stroke and therefore we have found a subspace distance measure most useful. To separate bleeding patients from the healthy ones and the patients with a clot it is necessary to use training data to determine a threshold level in the distance measure that separates the different classes. Once the threshold level has been determined a patient can be diagnosed. Experimental results from a phantom study shows promising results when it comes to separating the bleeding patients from the non-bleeding. We have also seen that the subspace distance potentially is a viable measure to determine the size of the bleeding. The results based on the clinical data are promising and indicates that this could be a viable approach and that further studies could be motivated. Results will be presented in the conference.

\section{CONCLUSIONS}

We have built an experimental prototype for microwave diagnostics of stroke patients. The aim is to separate bleeding strokes from healthy patients and patients with a blood clot. 10 antennas are mounted inside a helmet and worn by the patient during the examination. We are currently investigating two alternative signal processing algorithms, full dielectric reconstruction and a classifier algorithm. Initially we have received promising results with the classifier algorithm that motivates continued studies

\section{REFERENCES}

[1] B. Norrving, "Förekomst i befolkningen," Vårdalinstitutets Tematiska rum : Stroke - vård, omsorg och rehabilitering, 2007, [Online]. Available: http://www.vardalinstitutet.net.

[2] The National Board of Health and Welfare of Sweden, "National guidelines for stroke care 2005," Sweden, 2006. [Online]. Available: www.sos.se.

[3] S. Gabriel, R.W. Lau and C. Gabriel, "The dielectric properties of biological tissues: II. Measurements in the frequency range $10 \mathrm{~Hz}$ to 20 GHz," Phys. Med. Biol., vol. 41, pp. 2251-2269, 1996.

[4] H. Trefna, M. Persson, "Antenna array design for brain monitoring," Antennas and Propagation Society International Symposium, AP-S 2008, San Diego, CA, 5-11, July, 2008.

[5] D. Ireland, M. Bialkowski, "Feasibility study on microwave stroke detection using a realistic phantom and the FDTD method," Microwave Conference Proceedings (APMC), 2010 Asia-Pacific, pp. 1360 - 1363, Dec. 2010.

[6] S. Y. Semenov and D. R. Corfield, "Microwave Tomography for Brain Imaging: Feasibility Assessment for Stroke Detection," International Journal of Antennas and Propagation, vol. 2008, Article ID 254830, 2008

[7] A. Fhager, P. Hashemzadeh and M. Persson, "Reconstruction quality and spectral content of an electromagnetic time-domain inversion algorithm," IEEE Trans. Biomed. Eng., vol. 53, no. 8, pp. 1594-1604, Aug. 2006.

[8] M.A. Khorshidi, T. McKelvey, M. Persson, H. Dobsicek Trefna, "Classification of Microwave Scattering Data based on a Subspace Distance with Application to Detection of Bleeding Stroke," The Third International Workshop on Computational Advances in Multi-Sensor Adaptive Processing (CAMSAP 2009), Aruba, Dutch Antilles, 13-16 December 2009. 